Communications in Mathematical Physics manuscript No.

(will be inserted by the editor)

\title{
Multi-scaling of the $n$-point density function for coalescing Brownian motions
}

\author{
Ranjiva Munasinghe ${ }^{1 \star}$, R. Rajesh ${ }^{2 \star \star}$, Roger Tribe $^{1 \star \star \star}$, Oleg Zaboronski ${ }^{1 \dagger}$ \\ 1 Mathematics Institute, University of Warwick, Gibbet Hill Road, Coventry CV4 7AL, UK \\ 2 Institute of Mathematical Sciences, CIT Campus, Taramani, Chennai-600113, India
}

September 17, 2018

\begin{abstract}
This paper gives a derivation for the large time asymptotics of the $n$-point density function of a system of coalescing Brownian motions on $\mathbf{R}$.
\end{abstract}

\section{Introduction and statement of the main result}

The single species reaction-diffusion systems $A+A \rightarrow A$ (coalescence) and $A+A \rightarrow 0$ (annihilation) have been studied extensively in recent times 6, 7, 8, 9, 10, 11, 12, 13. A common interest in these papers is the departure from mean field behaviour and the computation of exact long-term asymptotics for the particle density.

Recently, it was predicted in [14] that the large time asymptotics for the probability $P_{t}(n, \Delta V)$ of finding $N$ particles in a fixed volume $\Delta V$ :

$$
P_{t}(n, \Delta V) \sim \begin{cases}t^{-\frac{n}{2}-\frac{n(n-1)}{4}} & d=1 \\ \left(\frac{\ln t}{t}\right)^{n}(\ln t)^{-\frac{n(n-1)}{2}} & d=2 \\ t^{-n} & d>2\end{cases}
$$

Note that the predictions for $d>2$ agree with mean field behaviour. The second part of the exponent in $d=2$ reflects multi-scaling, or deviation from linear scaling. In $d=2$ the multi-scaling is manifested in the second logarithmic term. This type of scaling is indicative of particles being anti-correlated 14. These predictions were obtained by use of the dynamical perturbative renormalization group methods in a field theoretic setting [6. The setting here is for finite rate reactions, leading to annihilations of randomly walking particles on a fixed lattice. After renormalization the large time limit rate tends to an instantaneous reaction 6. 13. Moreover the predictions carry over to the coalescing case, since they have the same effective field theory [1,8].

\footnotetext{
^ Electronic address: ranm@maths.warwick.ac.uk

$\star \star$ Electronic address: rrajesh@imsc.res.in

$\star \star \star$ Electronic address: tribe@maths.warwick.ac.uk

$\dagger$ Electronic address: olegz@maths.warwick.ac.uk
} 
The aim of this paper is to verify the conjectures of [14] in dimension $d=1$, where detailed probabilistic tools are available. We consider a system of coalescing Brownian particles on the real line. Each particle evolves independently until it collides with another particle, at which time the two colliding particles instantaneously coalesce into one. The $n$-point density function is defined, for distinct $y_{1}, \ldots, y_{n}$, by

$$
\left.P \text { [there exist particles in } d y_{1}, \ldots, d y_{n} \text { at time } t\right]=\rho_{n}\left(y_{1}, \ldots, y_{n} ; t\right) d y_{1} \ldots d y_{n} .
$$

Note that $\rho_{n}$ depends on the initial particle distribution. The existence of such a density is dicussed in the appendix.

Our main result can be stated precisely as the following asymptotic:

$$
\rho_{n}\left(y_{1}, \ldots, y_{n} ; t\right) \sim t^{-\alpha(n)} \prod_{1 \leq i<j \leq n}\left|y_{i}-y_{j}\right|
$$

where $\alpha(n)=n / 2+n(n-1) / 4$. This asymptotic has the meaning that the left hand side is bounded above and below by constant multiples $C_{1}, C_{2}$ of the right hand side. The upper bound is not actually an asymptotic, in that it holds simultaneously for all initial conditions, for all $t>0$ and for all $\left|y_{i}\right| \leq L t^{1 / 2}$, with a constant $C_{2}(L, n)<\infty$ depending only on $n$ and $L$. The lower bound for all $t \geq t_{0}$ and all $\left|y_{i}\right| \leq L t^{1 / 2}$, with a constant $C_{1}\left(n, L, t_{0}\right)>0$ that depends on $n, L, t_{0}$ and also on the initial condition. For the lower bound, the initial condition must satisfy a mild non-degeneracy condition; in particular it holds for deterministic initial conditions provided the gap between successive particles is bounded, but also if we assume the set of initial positions of particles $\left\{X_{0}^{i}: i \geq 1\right\}$ is non-zero, translationally invariant and spatially ergodic, in the sense that the distribution of $\left\{X_{0}^{i}: i \geq 1\right\} \cap(-\infty, 0]$ and $\left\{X_{0}^{i}: i \geq 1\right\} \cap[L, \infty)$ become independent as $L \rightarrow \infty$.

The key tool is the Karlin-McGregor formula for the non-coincidence probabilities for Brownian motions. Useful upper and lower bounds on this transition density, which already display the key anomalous

scaling term $t^{-\alpha(n)}$, are developed in section [3 by exploiting a representation known as the HarishChandra-Itzykson-Zuber formula (developed for random matrix problems).

The empty interval method, and its generalizations, have been used to derive expressions for higher order correlation functions in 7,8,9 for one-dimensional systems with instantaneous reactions. Large time asymptotics for the $n$-point correlation function density for the coalescing case with Poissonian initial conditions are found in [9], while the corresponding results for the annihilating case are given in 8. It was shown in this special case that the $n$-point density correlation function for the two systems are the same apart from the amplitude. This set of exact results was used to test the predictions (1.1) in [14. The large time scaling of the formulae for the $n$-point density functions given in 8,9 are not obvious as they involve a large combinatorial sum of terms with alternating signs.

The problem of deriving rigourously the logarithmic corrections (1.1) to the mean field answers in dimension $d=2$ remains open. It would also be interesting to find out if there is a natural multi-fractal interpretation of the multi-scaling. Another simple system for which RG calculations predict multi-scaling in the stationary state is the system of aggregating massive point clusters with stationary source of light particles. This system is relevant to turbulence, see [15]. It would be interesting to generalize the methods of present paper to prove multi-scaling for such cluster-cluster aggregation.

\section{Proof of the main result}

The proof is based on the following two lemmas. The first is a bound on the Karlin-McGregor formula for the transition density for non-intersecting Brownian motions [5] 3. Fix $x=\left(x_{1}, \ldots, x_{n}\right) \in \mathbf{R}^{n}$ and let $\left(X^{x_{i}}: i=1, \ldots, n\right)$ be independent Brownian motions with $X_{0}^{x_{i}}=x_{i}$. For $y \in \mathbf{R}^{n}$, denote by $G_{t}^{K M}(x, y)$ the density of the probability measure

$$
P\left[X_{t}^{x_{i}} \in d y_{i} \text { and the paths }\left(X_{s}^{x_{i}}: s \in[0, t]\right) \text { are non-intersecting for } i=1, \ldots, n .\right] .
$$


Then the Karlin-McGregor formula is

$$
G_{t}^{K M}(x, y)=\left|\begin{array}{ccc}
G_{t}\left(x_{1}, y_{1}\right) & \ldots & G_{t}\left(x_{1}, y_{n}\right) \\
\vdots & & \vdots \\
G_{t}\left(x_{n}, y_{1}\right) & \ldots & G_{t}\left(x_{n}, y_{n}\right)
\end{array}\right|
$$

where $G_{t}(a, b)=(2 \pi t)^{-1 / 2} e^{-|a-b|^{2} / 2 t}$ is the one dimensional Brownian transition density. Note that the function $G_{t}^{K M}(x, y)$ is the transition density for an $n$-dimensional Brownian motion killed on the set $\cup_{i \neq j}\left\{y_{i}=y_{j}\right\}$. One can check directly that the determinant expression (2.1) satisfies the heat equation with zero Dirichlet boundary conditions on this boundary, and that $\lim _{t \downarrow 0} G_{t}^{K M}(x, y)=\delta_{x=y}$.

The following lemma, proved in section 3] gives usable bounds on $G_{t}^{K M}(x, y)$.

Lemma 1 For all $x_{1}<\ldots<x_{n}, y_{1}<\ldots<y_{n}$ and $t>0$

$$
c_{n} \prod_{i=1}^{n} G_{t}\left(x_{i}, y_{n-i+1}\right) \leq \frac{G_{t}^{K M}(x, y)}{\Delta\left(x t^{-1 / 2}\right) \Delta\left(y t^{-1 / 2}\right)} \leq c_{n} \prod_{i=1}^{n} G_{t}\left(x_{i}, y_{i}\right),
$$

where $c_{n}^{-1}=\prod_{i=1}^{n} i$ ! and $\Delta(x)$ is the Vandermonde determinant defined by

$$
\Delta(x)=\Delta\left(x_{1}, \ldots, x_{n}\right)=\prod_{1 \leq i<j \leq n}\left(x_{i}-x_{j}\right) .
$$

The second lemma, proved in section 4 gives a simple upper bound on the $n$-point correlation function, which reflects the intuition that particles should be anti-correlated, in that the presence of a particle in $d x$ decreases the likelihood that there is a particle at another point $d y$.

Lemma 2 For any initial distribution of particles, the n-point density function satisfies

$$
\rho_{n}\left(y_{1}, \ldots, y_{n} ; t\right) \leq(\pi t)^{-n / 2} \text { for all } y_{i} \in \mathbf{R} \text { and } t>0 .
$$

The lower bound in the asymptotic (1.2) follows quickly from the lower bound on the KarlinMcGregor formula (2.2). Indeed, list the set of initial positions of particles as $\left\{X_{0}^{i}: i \geq 1\right\}$ and let $\Omega_{0}(t)$ be the event that there exist particles $X_{0}^{i_{1}}, \ldots, X_{0}^{i_{n}}$ satisfying

$$
X_{0}^{i_{k}} \in\left[2 k t^{1 / 2},(2 k+1) t^{1 / 2}\right] \text { for } k=1, \ldots, n .
$$

Then, applying the Karlin-McGregor formula between the points $X_{0}^{i_{1}}, \ldots, X_{0}^{i_{n}}$ and $y_{1}, \ldots, y_{n}$ we find, for $t \geq 1$,

$$
\begin{aligned}
& P\left[\text { there exist particles at } d y_{1}, \ldots, d y_{n} \text { at time } t\right] \\
& \quad \geq E\left[\mathbf{I}\left(\Omega_{0}(t)\right) G_{t}^{K M}\left(\left(X_{0}^{i_{1}}, \ldots, X_{0}^{i_{n}}\right),\left(y_{1}, \ldots, y_{n}\right)\right)\right] \\
& \quad \geq c_{n} E\left[\mathbf{I}\left(\Omega_{0}(t)\right) \Delta\left(X_{0}^{i_{1}} t^{-1 / 2}, \ldots, X_{0}^{i_{n}} t^{-1 / 2}\right) \prod_{k=1}^{n} G_{t}\left(X_{0}^{i_{k}}, y_{n-k+1}\right)\right] \Delta\left(y t^{-1 / 2}\right) d y_{1} \ldots d y_{n} \\
& \quad \geq C(n, L) t^{-n / 2} P\left[\Omega_{0}(t)\right] \Delta\left(y t^{-1 / 2}\right) d y_{1} \ldots d y_{n}
\end{aligned}
$$

where we have used (2.3) and $\left|y_{j}\right| \leq L t^{1 / 2}$ in the final inequality. We have also used $C(n, L, \ldots)$ to denote a finite non-zero quantity, depending only on the quantities listed, but whose exact value is unimportant and may change from line to line. It remains only to bound $P\left[\Omega_{0}(t)\right]$ from below, independently of $t \geq t_{0}$. This clearly holds under the two sets of assumptions described in section 1 and in particular for Poissonian initial conditions. 
For the upper bound in the asymptotic (1.2) we estimate the probability that there exist particles at $d y_{1}, \ldots, d y_{n}$ at time $2 t$ by conditioning on the set $\left\{X_{t}^{i}: i \in \mathbf{N}\right\}$ of positions of the particles at time $t$. For the desired particles to exist at time $2 t$, one of the events

$$
\Omega_{i_{1}, \ldots, i_{n}}(t)=\left\{X_{2 t}^{i_{k}} \in d y_{k} \text { and the paths }\left(X_{s}^{i_{k}}: s \in[t, 2 t]\right) \text { do not intersect for } k=1, \ldots, n\right\},
$$

for some $i_{1}<\ldots<i_{n}$, must occur. Applying the Markov property at time $t$ and the upper bounds in Lemma 1 we find, for all $t>0$,

$P$ [there exist particles at $d y_{1}, \ldots, d y_{n}$ at time $\left.2 t\right]$

$$
\begin{aligned}
& \leq \sum_{i_{i}<\ldots<i_{n}} P\left[\Omega_{i_{1}, \ldots, i_{n}}(t)\right] \\
& \leq c_{n} \sum_{i_{i}<\ldots<i_{n}} E\left[\Delta\left(X_{t}^{i_{1}} t^{-1 / 2}, \ldots, X_{t}^{i_{n}} t^{-1 / 2}\right) \prod_{k=1}^{n} G_{t}\left(X_{t}^{i_{k}}, y_{k}\right)\right] \Delta\left(y t^{-1 / 2}\right) d y_{1} \ldots d y_{n} \\
& \leq C(n)\left(\int_{\mathbf{R}^{n}} G_{t}(x, y) \Delta\left(x t^{-1 / 2}\right) \rho_{n}(x ; t) d x\right) \Delta\left(y t^{-1 / 2}\right) d y_{1} \ldots d y_{n} \quad \text { see (B.1) } \\
& \leq C(n, L) t^{-n / 2} \Delta\left(y t^{-1 / 2}\right) d y_{1} \ldots d y_{n} .
\end{aligned}
$$

The final inequality uses the substitution $x \rightarrow x t^{1 / 2}$ and the bound from Lemma 2

\section{Proof of Lemma 1, the Karlin-Macgregor bounds}

First note that Brownian scaling implies that $G_{t}^{K M}(x, y)=t^{-n / 2} G_{1}^{K M}\left(x t^{-1 / 2}, y t^{-1 / 2}\right)$, and we need only prove the lemma for $t=1$. Factoring out common terms from the rows in (2.1) we find that

$$
G_{1}^{K M}(x, y)=(2 \pi)^{-n / 2} \operatorname{det}(E(x, y)) \prod_{i=1}^{n} e^{-\left(x_{i}^{2}+y_{i}^{2}\right) / 2}
$$

where $E(x, y)$ is the matrix with entries $\exp \left(x_{i} y_{j}\right)$. We now use the Harish-Chandra-Itzykson-Zuber formula [2, which is widely used in random matrix theory. This states that

$$
\operatorname{det}(E(x, y))=c_{n} \Delta(x) \Delta(y) \int_{\mathbf{U}(n)} \exp \left(\operatorname{tr}\left(U X U^{\dagger} Y\right)\right) \mu(d U),
$$

where $\operatorname{tr}(A)$ is the trace of a matrix $A, \mu(d U)$ is (normalized) Haar measure on the unitary group $\mathbf{U}(n)$ and $X, Y$ are the diagonal matrices with entries $x_{1}, \ldots, x_{n}$ and $y_{1}, \ldots, y_{n}$. A short proof of this formula is found in [4] (appendix A.5).

The fact that $U$ is unitary ensures that the function $F(U)=\operatorname{tr}\left(U X U^{\dagger} Y\right)$ is real valued (recall that $U^{\dagger}$ is the conjugate transpose of $U$ so that $\left.U U^{\dagger}=U^{\dagger} U=I\right)$. By the compactness of $\mathbf{U}(n), F(U)$ achieves its maximum and minimum values and we claim that

$$
\min _{U \in \mathbf{U}(n)} F(U)=\sum_{i=1}^{n} x_{n-i+1} y_{i}, \quad \max _{U \in \mathbf{U}(n)} F(U)=\sum_{i=1}^{n} x_{i} y_{i} .
$$

Bounding the integral in the Harish-Chandra-Itzykson-Zuber formula using these maxima and minima in the integrand leads to the bounds (2.2).

It remains only to prove the claim (3.1), for which we will find all the stationary points of the smooth function $F$ on the manifold $\mathbf{U}(n)$. A matrix $V$ is in the tangent space to $\mathbf{U}(n)$ at the point $U$ precisely 
when $U V^{\dagger}+V U^{\dagger}=U^{\dagger} V+V^{\dagger} U=0$. The first derivative of $F$ in the direction of $V$ in the tangent space is given by

$$
\begin{aligned}
D_{V} F(U) & =\operatorname{tr}\left(U X V^{\dagger} Y\right)+\operatorname{tr}\left(V X U^{\dagger} Y\right) \\
& =\operatorname{tr}\left(V^{\dagger} Y U X\right)+\operatorname{tr}\left(V X U^{\dagger} Y\right) \quad(\text { since } \operatorname{tr}(A B)=\operatorname{tr}(B A) \text { for Hermitian } B) \\
& =\operatorname{tr}\left(U V^{\dagger} Y U X U^{\dagger}\right)+\operatorname{tr}\left(V X U^{\dagger} Y\right) \quad\left(\text { since } \operatorname{tr}\left(U A U^{\dagger}\right)=\operatorname{tr}(A)\right) \\
& =-\operatorname{tr}\left(V U^{\dagger} Y U X U^{\dagger}\right)+\operatorname{tr}\left(V U^{\dagger} U X U^{\dagger} Y\right) \quad\left(\text { using } U V^{\dagger}=-V U^{\dagger}\right) \\
& =\operatorname{tr}\left(V U^{\dagger}\left[U X U^{\dagger}, Y\right]\right)
\end{aligned}
$$

(where $[A, B]=A B-B A$ is the commutator). The map $V \rightarrow V U^{\dagger}$ bijectively maps the tangent space onto the Lie group Lie $(\mathbf{U}(n))$ defined by $\left\{W: W^{\dagger}+W=0\right\}$. It is straightforward to check that $\operatorname{tr}(W A)=0$ for all $W \in \operatorname{Lie}(\mathbf{U}(n))$ implies that $A=0$. The vanishing of $D_{V} F(U)$ therefore implies that $\left[U X U^{\dagger}, Y\right]=0$. This in turn implies, since $Y$ is diagonal with distinct eigenvalues, that $U X U^{\dagger}$ is a diagonal matrix. The matrix $U X U^{\dagger}$ has the same distinct eigenvalues as $X$, so that the entries on the diagonal of $U X U^{\dagger}$ must be a permutation of $x_{1}, \ldots, x_{n}$. Hence the value of $F(U)$ at a stationary point is $\sum_{i=1}^{n} x_{\pi(i)} y_{i}$ for some permutation $\pi$. We must show that this value is maximized when $\pi=$ Id the identity permutation and minimized at the involution $\pi(i)=N+1-i$. Argue by contradiction and suppose that $\pi \rightarrow \sum_{i=1}^{n} x_{\pi(i)} y_{i}$ is maximized over permutations at some $\pi_{0} \neq \mathrm{Id}$. Then there exist $i<j$ for which $\pi_{0}(i)>\pi_{0}(j)$. Let $\pi^{(j k)}$ be the transposition permutation, swapping the $j$ th and $k$ th elements. Then an increased value is found by taking the composition $\pi_{1}=\pi^{(j k)} \circ \pi_{0}$ permutation, since

$$
\sum_{i=1}^{n} x_{\pi_{1}(i)} y_{i}-\sum_{i=1}^{n} x_{\pi_{0}(i)} y_{i}=\left(x_{\pi_{0}(i)}-x_{\pi_{0}(j)}\right)\left(y_{j}-y_{i}\right)>0
$$

using the ordering $x_{1}<\ldots<x_{n}, y_{1}<\ldots<y_{n}$. The minimum follows from a similar argument.

\section{Proof of Lemma 2, the $n$-point density bound}

We start with a simple construction of coalescing particles as follows. Fix $x_{1}<x_{2}<\ldots$. Take I.I.D. Brownian motions $\left(B^{i}: i \geq 1\right)$ and set $\hat{X}_{t}^{x_{i}}=x_{i}+B_{t}^{i}$, for $t \geq 0$, the non-interacting Brownian paths. Then define $X_{t}^{x_{1}}=\hat{X}_{t}^{x_{1}}$ and inductively for $i \geq 2$

$$
X_{t}^{x_{i}}= \begin{cases}\hat{X}_{t}^{x_{i}} & \text { for } t \leq \tau\left(x_{i}, x_{i-1}\right) \\ X_{t}^{x_{i-1}} & \text { for } t>\tau\left(x_{i}, x_{i-1}\right)\end{cases}
$$

where $\tau\left(x_{i}, x_{i-1}\right)=\inf \left\{t: \hat{X}_{t}^{x_{i}}=X_{t}^{x_{i-1}}\right\}=\inf \left\{t: X_{t}^{x_{i}}=X_{t}^{x_{i-1}}\right\}$. The path $t \rightarrow X_{t}^{x}$ gives the position of the particle that started at $x$. This construction shows that the process starting from infinitely many particles can be approximated by the process starting with finitely many particles in an increasing way. It will therefore be sufficient to bound $\rho_{n}$ over all finite starting positions. We write $P_{x_{1}, x_{2}, \ldots}$ to indicate the starting positions.

The fact that $\rho_{1}(y) \leq(\pi t)^{-1 / 2}$, for any initial particle configuration, is well known. In section 9 of [16] it states that the time reversal duality formula for coalescing Brownian motions for $a<b$ is given by

$$
P_{x_{1}, \ldots, x_{m}}\left[\left\{X_{t}^{x_{i}}: i \leq m\right\} \cap[a, b] \neq \emptyset\right]=P_{a, b}\left[\left[X_{t}^{a}, X_{t}^{b}\right] \cap\left\{x_{1}, \ldots, x_{m}\right\} \neq \emptyset\right] .
$$

We can take the supremum of the above duality over all starting configurations to obtain

$$
\sup _{x_{i}} P_{x_{1}, \ldots, x_{m}}\left[\left\{X_{t}^{x_{i}}: i \geq 1\right\} \cap[a, b] \neq \emptyset\right]=P_{a, b}\left[X_{t}^{a}<X_{t}^{b}\right]
$$

which can be explicitly calculated and is bounded by $(\pi t)^{-1 / 2}(b-a)$. We give an elementary approach to this bound that avoids duality in Appendix $\mathrm{A}$ 
For the extension to $\rho_{n}$ given in Lemma 2 we fix $a_{1}<b_{1}<a_{2}<\ldots<a_{n}<b_{n}$. Define

$$
\Omega_{j}=\left\{\text { there exists a particle in }\left[a_{j}, b_{j}\right] \text { at time } t\right\} .
$$

We shall show by induction that

$$
P_{x_{1}, \ldots, x_{m}}\left[\Omega_{1} \cap \ldots \cap \Omega_{n}\right] \leq \prod_{i=1}^{n} \frac{\left(b_{i}-a_{i}\right)}{\pi t^{1 / 2}} .
$$

Decompose $\Omega_{1}$ as the disjoint union $\Omega_{1}=\cup_{k} \Omega_{1, k}$ where

$$
\Omega_{1, k}=\left\{X_{t}^{x_{i}}<a_{1} \text { for } i=1, \ldots, k-1 \text { and } X_{t}^{x_{k}} \in\left[a_{1}, b_{1}\right]\right\} .
$$

Then conditional on $\Omega_{1, k}$ the processes $\left(X^{x_{i}}: i \geq k+1\right.$ ) evolve as coalescing Brownian motions with an extra lower absorbing boundary along the path $t \rightarrow X_{t}^{x_{k}}$. The construction above shows that this extra absorbing path only lowers the probability that any of the paths $\left(X^{x_{i}}: i \geq k+1\right)$ reach the intervals $\left[a_{2}, b_{2}\right], \ldots,\left[a_{n}, b_{n}\right]$. Thus, applying Bayes formula,

$$
\begin{aligned}
P_{x_{1}, \ldots, x_{m}}\left[\Omega_{1} \cap \ldots \cap \Omega_{n}\right] & =\sum_{k=1}^{m} P_{x_{1}, \ldots, x_{m}}\left[\Omega_{2} \cap \ldots \cap \Omega_{n} \mid \Omega_{1, k}\right] P_{x_{1}, \ldots, x_{m}}\left[\Omega_{1, k}\right] \\
& \leq \sum_{k=1}^{m} P_{x_{k+1}, \ldots, x_{m}}\left[\Omega_{2} \cap \ldots \cap \Omega_{n}\right] P_{x_{1}, \ldots, x_{m}}\left[\Omega_{1, k}\right] \\
& \leq \sum_{k=1}^{m} P_{x_{1}, \ldots, x_{m}}\left[\Omega_{2} \cap \ldots \cap \Omega_{n}\right] P_{x_{1}, \ldots, x_{m}}\left[\Omega_{1, k}\right] \\
& =P_{x_{1}, \ldots, x_{m}}\left[\Omega_{2} \cap \ldots \cap \Omega_{n}\right] P_{x_{1}, \ldots, x_{m}}\left[\Omega_{1}\right] .
\end{aligned}
$$

and the result (4.11), and hence Lemma 2 follows.

\section{A. An elementary approach to the one point density}

This elementary approach may be of some interest for systems that do not have a duality relation. Fix $a \in[0,1]$ and let $p\left(x_{1}, \ldots, x_{n}\right)$ be the probability that, starting some coalescing Brownian motions $x_{1}, \ldots, x_{n}$, there is a particle at time $t$ in the interval $[0, a]$. We aim to show, by induction on $n$, that

$$
p\left(x_{1}, \ldots, x_{n}\right) \leq C t^{-1 / 2} a
$$

for some constant $C<\infty$. We suppose $x_{1}<\ldots<x_{n+1}$. Condition on the path $t \rightarrow X_{t}^{(n+1)}$ of the path started at $x_{n+1}$. We can consider three cases: if $X_{t}^{(n+1)}<0$ then we are sunk since no other path can ever enter $[0, a]$ due to coalescence; if $X_{t}^{(n+1)} \in[0, a]$ then we are done; finally if $X_{t}^{(n+1)}>a$ then we still need some of the particles from $x_{1}, \ldots, x_{n}$ to enter $[0, a]$, but there is an absorbing boundary along the path of $X_{t}^{(n+1)}$. This absorbing boundary can only lower the chance of getting particles where we want. This splitting of possibilities leads to

$$
p\left(x_{1}, \ldots, x_{n+1}\right) \leq C t^{-1 / 2} a P\left[X^{(n+1)}>a\right]+P\left[X^{(n+1)} \in[0, a]\right] .
$$

It is straightforward to find $c_{0}<\infty$ so that for a Brownian motion $\left(X_{t}\right)$, and for $L \geq 1, t>0$,

$$
\frac{P\left[X_{t} \in[0, a] \mid X_{0}=x\right]}{P\left[X_{t}<a \mid X_{0}=x\right]} \leq c_{0} L a t^{-1 / 2} \quad \text { whenever }|x| \leq L t^{-1 / 2}
$$

Suppose now that $x_{i} \in\left[-L t^{1 / 2}, L t^{1 / 2}\right]$ for all $i$. Using this bound the induction argument works with the choice $C=c_{0} L$. In particular we have shown that

$$
\sup _{\left|x_{i}\right| \leq L t^{1 / 2}} p\left(x_{1}, \ldots, x_{m}\right) \leq c_{0} L t^{-1 / 2} a .
$$


Applying this with the choice $L=O(\sqrt{\log (t)})$, and using a simple bound that any particle starting outside $\left[-L t^{1 / 2}, L t^{1 / 2}\right]$ can reach the intervals $[0, a]$ (we need only control particles starting exactly at $\pm L t^{1 / 2}$ by coalescence) we find that

$$
\sup _{x_{i}} p\left(x_{1}, \ldots, x_{m}\right) \leq C t^{-1 / 2}(\log (t))^{1 / 2} a
$$

where the supremum is over all possible initial configurations. One can remove the unwanted logarithm term by a blocking argument, but this is already longer than the elegant duality argument, which has the extra advantage of achieving the optimal constant.

\section{B. A note on the existence of $\rho_{n}$}

We briefly consider for which measure $\rho_{n}(x ; t)$ acts as a density. List the (disjoint) positions of the particles at time $t$ as $\left\{X_{t}^{i}: i \geq 1\right\}$. Define a random measure $\mu_{t}^{n}$ on $\mathbf{R}^{n}$ by

$$
\mu_{t}^{n}=\sum_{i_{1}} \sum_{i_{2} \neq i_{1}} \ldots \sum_{i_{n} \neq i_{1}, \ldots, i_{n-1}} \delta\left(X_{t}^{i_{1}}, \ldots, X_{t}^{i_{n}}\right)
$$

where $\delta(x)$ is a point mass at $x$. Let $C_{n}(\delta)$ denote the cube $[0, \delta)^{n}$. The cubes $k+C_{n}\left(2^{-m}\right)$, for $k \in$ $\left(2^{-m} \mathbf{Z}\right)^{n}$, partition $\mathbf{R}^{n}$. Take $A \subseteq \mathbf{R}^{n}$ open. For an initial condition with only finitely many particles, the measure $\mu_{t}^{n}$ has only finitely many atoms which do not lie on $\cup_{i \neq j}\left\{x_{i}=x_{j}\right\}$. Therefore we have the increasing limit

$$
\lim _{m \rightarrow \infty} \sum_{k \in\left(2^{-m} \mathbf{Z}\right)^{n}} \mathbf{I}\left(\mu_{t}^{n}\left(k+C_{n}\left(2^{-m}\right)\right)>0\right)=\mu_{t}^{n}(A)
$$

where the sum over $k$ is restricted to those terms for which $k_{1}, \ldots, k_{n}$ are distinct. The event $\mu_{t}^{n}(k+$ $\left.C_{n}\left(2^{-m}\right)\right)>0$ can be rewritten as $\left[k_{j}, k_{j}+2^{-m}\right] \cap\left\{X_{t}^{i}: i \geq 1\right\} \neq \emptyset$ for $j=1, \ldots, n$. Taking expectations and applying (4.1) we find that $E\left[f d \mu_{t}^{n}\right] \leq(2 \pi t)^{-n / 2} \int f d x$ when $f=\mathbf{I}(A)$. By an approximation argument this also holds for all measurable $f$ and by monotonicity it holds for any initial distribution of particles. We may then define $\rho_{n}(x ; t)=\rho\left(x_{1}, \ldots, x_{n} ; t\right)$ as the density of the measure $E\left[\mu_{t}^{n}\right]$, that is the Radon Nicodym derivative with respect to Lebesgue measure, so that

$$
\int_{\mathbf{R}^{n}} f(x) \rho_{n}(x ; t) d x=E\left[\sum_{i_{1}} \sum_{i_{2} \neq i_{1}} \ldots \sum_{i_{n} \neq i_{1}, \ldots, i_{n-1}} f\left(X_{t}^{i_{1}}, \ldots, X_{t}^{i_{n}}\right)\right] .
$$

The Lebesgue differentiation theorem implies that $\rho(x ; t)=\lim _{\delta \rightarrow 0} \delta^{-n} E\left[\mu_{t}^{n}\left(x+C_{n}(\delta)\right]\right.$ for almost all $x$. Choosing $x_{1}, \ldots, x_{n}$ disjoint we obtain

$$
\rho\left(x_{1}, \ldots, x_{n} ; t\right)=\lim _{\delta \rightarrow 0} \delta^{-n} E\left[N_{t}\left(\left[x_{1}, x_{1}+\delta\right]\right) \ldots N_{t}\left(\left[x_{n}, x_{n}+\delta\right]\right)\right]
$$

where $N_{t}(A)$ is the number of particles inside $A$ at time $t$. However we claim we may replace $N_{t}\left(\left[x_{1}, x_{1}+\delta\right]\right)$ by the indicator $\mathbf{I}\left(\left[x_{1}, x_{1}+\delta\right]\right.$ contains a particle $)$ in this limit. The error when doing this occurs if there are two or more particles in $\left[x_{1}, x_{1}+\delta\right]$ and is dominated by $E\left[\mu_{t}^{n+1}\left(\left(x_{1}, x_{1}, x_{2}, \ldots, x_{n}\right)+C_{n+1}(\delta)\right)\right]$. This error is therefore of order $\delta^{n+1}$, so that replacing $N_{t}\left(\left[x_{i}, x_{i}+\delta\right]\right)$ in this way for each $i=1, \ldots, n$ we reach the usual definition of $\rho_{n}(x ; t)$ as, for distinct $x_{1}, \ldots, x_{n}$,

$$
\rho\left(x_{1}, \ldots, x_{n} ; t\right)=\lim _{\delta \rightarrow 0} \delta^{-n} P \text { [all the intervals }\left[x_{i}, x_{i}+\delta\right] \text { for } i=1, \ldots, n \text { are non-empty } .
$$




\section{References}

1. Hinrichsen, H.: Nonequilibrium Critical Phenomena and Phase Transitions into Absorbing States. arXiv: cond-mat/0001070 May 22, 2000.

2. Itzykson, C., Zuber, J.B.: The planar approximation. II. J. Math. Phys. 21, 411 (1980).

3. Bohm, W., Mohanty, S.G.: On the Karlin-McGregor Theorem \& Applications. The Annals of Applied Probability, Vol. 7, No. 2, 314-325 (1997).

4. Mehta, M.L.: Random Matrices. $2^{\text {nd }}$ edition, Academic Press, New York, 1991

5. Karlin, S. and McGregor, J.G.: Coincidence Probabilities. Pacific J. Math. 9, 1141-1164 (1959).

6. Cardy, J.: Field Theory and Nonequilibrium Statistical Mechanics. Available from http://www-thphys.physics.ox.ac.uk/users/JohnCardy/

7. ben-Avraham, D., Masser, T.O.: Method of intervals for the study of diffusion-limited annihilation, A+A $\rightarrow 0$. Phys Rev E, 63, 066108 (2001).

8. ben-Avraham, D., Masser, T.O.: Correlation functions for diffusion-limited annihilation, A+A $\rightarrow 0$. arXiv: cond-mat/0106306 v2, August 13, 2001.

9. ben-Avraham, D.: Complete Exact solution of Diffusion Limited Coalescenece, A+A $\rightarrow$ A. arXiv: cond-mat/9803281 v1, March 23, 1998.

10. Doering, C.R.: Microscopic spatial correlations induced by external noise in a reaction-diffusion system. Physica A 188, 386 (1992).

11. Bramson, M., Lebowitz, J.L.: Asymptotic behaviour of diffusion-dominated annihilation reactions. Phys Rev Lett 61 21 (1988).

12. Howard, M.J.: J Phys A 293437 (1996)

13. Lee, B.P.: Renormalization Group Calculation for the reaction kA $\rightarrow \emptyset$, J. Phys. A 27 2633-2652 (1994).

14. Munasinghe, R.M., Rajesh, R., Zaboronski, O.V.: Multi-Scaling of correlation functions in single species Reactiondiffusion systems. arXiv: cond-mat/0506398 June 16, 2005.

15. Connaughton, C., Rajesh, R., Zaboronski, O.V.: Breakdown of Kolmogorov Scaling in Models of Cluster Aggregation. Phys. Rev. Lett 94, 194503 (2005).

16. Donnelly, P., Evans, S., Fleischmann, K., Kurtz, T.G., Zhou, X.: Continuum-sites stepping-stone models, coalescing exchangeable partitions, and random trees. Ann. Prob. 28, 1063-1110, (2000) .

17. Rogers, L.C.G., Williams, D.: Markov Processes and Maritngales Volume 2: Ito Calculus. Cambridge: Cambridge University Press 1994

18. Xiong, J., Zhou, X.: On the duality between coalescing Brownian motions. Canad. J. Math. 57 (1), $204-224$ (2005). 